\title{
Relationship of the Medial Clavicular Head to the Manubrium in Normal and Symptomatic Degenerated Sternoclavicular Joints
}

\author{
Alexander Van Tongel, MD, Jens Valcke, Msc, Iwein Piepers, Msc, Thomas Verschueren, MD, and Lieven De Wilde, MD, PhD \\ Investigation performed at the Department of Orthopaedic Surgery and Traumatology, Ghent University Hospital, Ghent, Belgium
}

\begin{abstract}
Background: Clavicular prominence is common in patients with symptomatic degenerative sternoclavicular arthritis. It is unclear if this is caused by enlargement or subluxation of the clavicle. The aim of this report is to describe a reproducible measurement technique to evaluate the relationship of the medial clavicular head to the manubrium.
\end{abstract}

Methods: One hundred normal sternoclavicular joints, twenty-five sternoclavicular joints with symptomatic degenerative arthritis, and twenty-five non-symptomatic sternoclavicular joints on the contralateral side were studied with three-dimensional (3D) reconstruction with use of computer modeling. The greatest width (anterior-posterior distance) and height (superiorinferior distance) of the clavicle in the sagittal plane were measured, and the positions of the anterior and superior borders of the medial clavicle and their distances to the frontal and axial planes, respectively, were evaluated. The ratio of the anteriorposterior distance to the anterior-frontal plane distance was measured to evaluate the anterior-posterior position of the clavicle and the ratio of the superior-inferior distance to the superior-axial plane distance was measured to evaluate its superoinferior position. If the ratio was not in the $95 \%$ normal range, the clavicle was defined as subluxated. The reproducibility of this technique was evaluated on the basis of the interobserver and intraobserver reliability.

Results: This technique showed good interobserver and intraobserver reliability. The mean anterior-posterior and superiorinferior distances were significantly larger in association with symptomatic sternoclavicular arthritis than in the normal sternoclavicular joints ( $p$ 0001). The clavicle was subluxated anteriorly in twenty-two of the twenty-five cases of symptomatic sternoclavicular arthritis, but it was not subluxated superiorly.

Conclusions: The medial clavicular head in patients with degenerative sternoclavicular arthritis is significantly larger than it is in the normal population, and it is usually subluxated anteriorly.

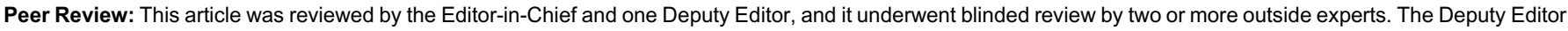

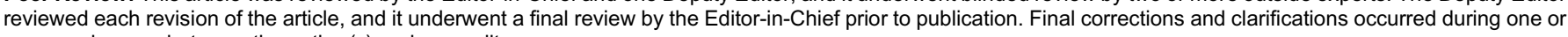
more exchanges between the author(s) and copyeditors.

S ymptomatic degenerative sternoclavicular arthritis is an infrequent condition ${ }^{1,2}$, and effective management is often hindered by a limited understanding of the anatomy and the pathology $y^{3}$. Often patients with this condition have swelling and/or mechanical pain at the sternoclavicular joint. Clinical examination sometimes reveals a prominent clavicle $e^{4-7}$. We are not aware of any studies that have evaluated whether this prominence is caused by osseous enlargement and osteophytes or by subluxation of the clavicle.
In the past, the bones forming a joint have been evaluated with two-dimensional (2D) imaging techniques. ${ }^{8}$. Three-dimensional (3D) computed tomography (CT) scan reconstruction improves the accuracy of the measurements. This technique can facilitate determination of the amount and direction of the joint subluxation $^{9,10}$.

The aim of this report is to describe a reproducible measurement technique to evaluate the relationship of the medial clavicular head to the manubrium in a normal population and in

Disclosure: None of the authors received payments or services, either directly or indirectly (i.e., via his or her institution), from a third party in support of any aspect of this work. One or more of the authors, or his or her institution, has had a financial relationship, in the thirty-six months prior to submission of this work, with an entity in the biomedical arena that could be perceived to influence or have the potential to influence what is written in this work. No author has had any other relationships, or has engaged in any other activities, that could be perceived to influence or have the potential to influence what is written in this work. The complete Disclosures of Potential Conflicts of Interest submitted by authors are always provided with the online version of the article. 

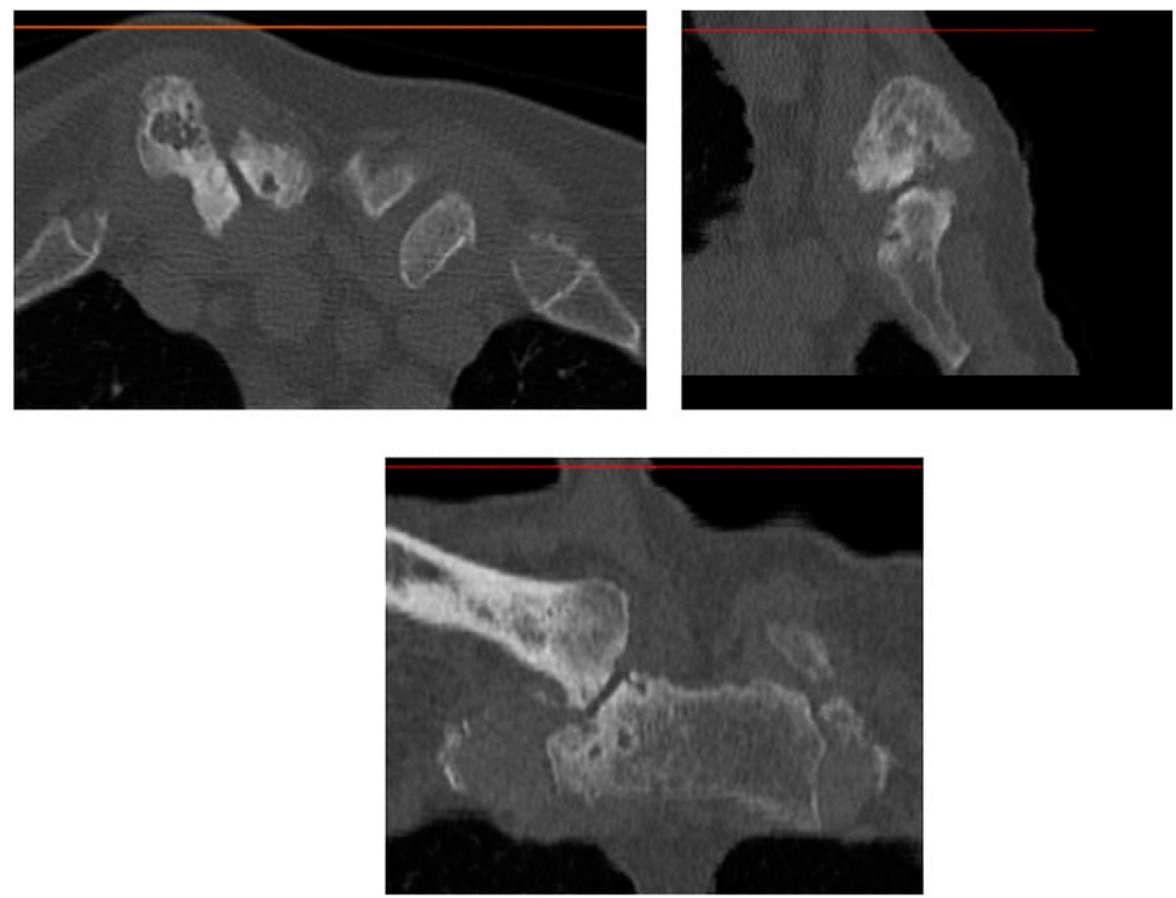

Fig. 1

Axial, sagittal, and frontal CT views of symptomatic degenerative sternoclavicular arthritis.

patients with painful prominence related to sternoclavicular arthritis. It was hypothesized that the prominence was caused by both an enlargement of the clavicle due to osteophytes and anteriorsuperior subluxation of the clavicle.

\section{Materials and Methods}

A pproval for this study was obtained from the local ethical committee (EC/ 2011-069).

Two groups of patients were evaluated: a group of young adult patients without any sign of pathology at the sternoclavicular joint (Group 1) and a group of patients with painful prominence related to sternoclavicular arthritis (Group 2).

Group 1 included patients between twenty-four and thirty-five years old (mean, 26.9 years old) for whom a CT scan that had been planned for evaluation of shoulder pathology showed no sternoclavicular pathology. Both shoulders and sternoclavicular joints were scanned simultaneously. Other pathological conditions of the sternoclavicular joint (traumatic or atraumatic instability, or inflammatory disease such as sternocostoclavicular hyperostosis or rheumatoid arthritis, osteitis condensans, or septic arthritis) were excluded by physical examination and on the basis of the patient's history. The CT scans were inspected for structural osseous lesions of the clavicle (e.g., cysts and visible osseous deformations of the clavicle) and if such lesions were present the subject was excluded. The final group of fifty subjects (twenty-five female and twenty-five male) included patients with glenohumeral instability (thirty), calcifying tendinitis (seven), frozen shoulder (five), and fracture of the proximal part of the humerus (eight).

Group-2 patients all had clinical signs of symptomatic sternoclavicular arthritis (mechanical pain at the sternoclavicular joint and swelling), confirmed on CT (showing bone cyst, osteophytes, and/or narrowing of the joint space) (Fig. 1). The mean age was 61.6 years. All patients were positioned in the CT gantry according to a previously described method, supine with a strap around the body and a cushion on the abdomen to keep the arm adducted in the coronal plane and the forearm flexed in the sagittal plane ${ }^{11}$. This standardized position mimics a reproducible surgical position and minimizes positional errors.

\section{Image Analysis and Interpretation}

A Somatom Volume Zoom CT scanner (Siemens, Erlangen, Germany) with a matrix set to $512 \AA \sim 512, \mathrm{kV}: 140 /$ eff. mAs: 350 was used. The field of view (FOV) was adapted to each individual patient. This resulted in a maximum of $500 \mathrm{~mm}$ for both shoulders and $180 \mathrm{~mm}$ for one shoulder with a pixel size of no more than 0.97 or $0.35 \mathrm{~mm}$, respectively. The glenohumeral and sternoclavicular joints were scanned with maximum $1.5-\mathrm{mm}$ interval slices.

Three independent investigators imported the Digital Imaging and Communications in Medicine (DICOM) CT images into medical imaging computer software (Mimics 14.12 for Intel $\times 86$ Platform V14.0.0.90 19922012; Materialise, Leuven, Belgium) to create 3D images of the sternoclavicular joint. Both bones of the sternoclavicular joint could be separated digitally and virtually manipulated.

An important part of this technique is the definition of the frontal plane. To our knowledge, this study is the first to describe a frontal plane as a best-fitting plane on an area on the manubrium (see Appendix). This consistent area appears to represent a structural adaptation of bone to mechanical loading as has been described in other bones ${ }^{12,13}$. This triangular area is based on the Y-shaped area that can be seen when placing the manubrium against a bright light or in a $3 \mathrm{D}$ CT-reconstructed manubrium. This area was described by Xiu et al. as the bare area that can be used to obtain autologous bone graft ${ }^{14}$. The attachment of the pectoralis major muscle covers the medial and lateral borders of the triangle ${ }^{14}$.

With the use of computer modeling (3-matic; Materialise), the best-fitting plane was determined according to this triangular zone (Fig. 2) and this plane was called the frontal plane. The axial plane is perpendicular to the frontal plane and passes through the highest points left and right of the presternal notch (suprasternal notch) (Fig. 3). Using the same computer modeling technique in the sagittal plane, we determined the intersection point of the most anterior (A) and posterior $(\mathrm{P})$ points of the medial clavicle with a plane parallel to the frontal plane. We also determined the intersection point of the most superior (S) and inferior (I) points of the medial clavicle with a plane parallel to the axial plane (Fig. 4).

Next, the distance between point A and point P (AP distance) was measured, as was the distance between point $\mathrm{A}$ and the frontal plane (AFr distance: positive if posterior to the frontal plane and negative if anterior to the 


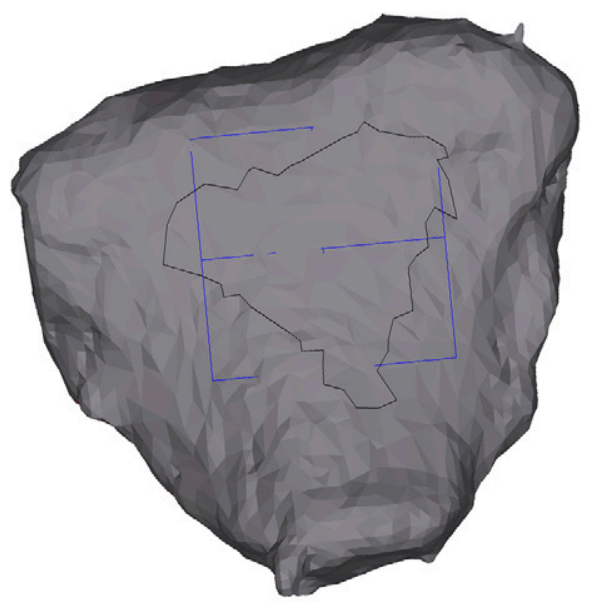

Fig. 2

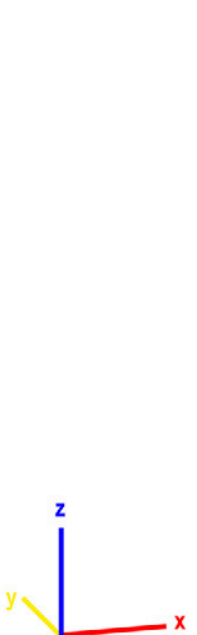

Fig. 3

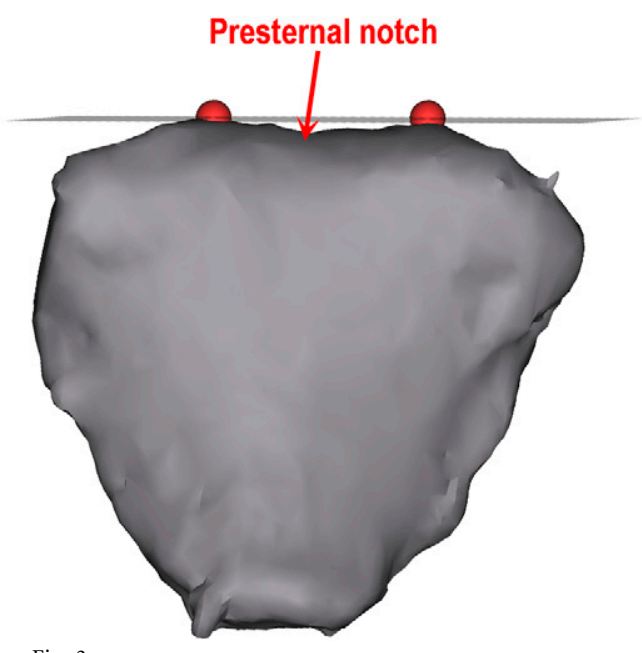

Fig. 2 Determination of the best-fitting plane based on a consistent triangular area in the manubrium. Fig. 3 Determination of the axial plane.

frontal plane) (Fig. 5). Also the distance between point $\mathrm{S}$ and point I (SI distance) was measured, as was the distance between point $S$ and the axial plane (SAx distance: positive if superior to the axial plane and negative if inferior to axial plane) (Fig. 6). This was done for all normal and abnormal sternoclavicular joints.

Next the AFr/AP and SAx/SI ratios were measured for all patients. These ratios were used to evaluate the position of the clavicle and to exclude the variability of the AP and SI distances. The $95 \%$ prediction interval for the AFr/ $\mathrm{AP}$ and $\mathrm{SAx} / \mathrm{SI}$ ratios was measured in the normal population. If the ratio of the clavicle of the sternoclavicular joint with symptomatic degenerative arthritis to the clavicle of the non-symptomatic, contralateral joint was not in this normal range, the clavicle was defined as subluxated anteriorly or posteriorly or as subluxated superiorly or inferiorly.

Intraobserver and interobserver reliability was calculated for all parameters. Two different examiners (A.V.T. and J.V.) determined all planes and points and measured all parameters on the scans of thirty joints (twenty normal, five on the arthritic side, and five on the non-arthritic, contralateral side). One examiner (A.V.T.) repeated the measurements with a time interval of fourteen days between measurements. The intraobserver and interobserver reliability was assessed with intraclass correlation coefficients (ICCs) on the basis of two-way random-effect models, with use of an absolute-agreement definition ${ }^{15}$.

To evaluate the correlation between the different parameters, the Student $t$ test was used for the normally distributed parameters. The MannWhitney $\mathrm{U}$ test was used for the not normally distributed parameters.

\section{Source of Funding}

There was no external funding source for this study.

\section{Results}

The intraobserver and interobserver ICCs were excellent for 1 all measured parameters (see Appendix).

The measured dimensions are listed in the Appendix.

In Group 1 (normal population), the AP distance of the clavicular head was greater in men than in women $(\mathrm{p}<0.001)$. There was no significant difference between the men and women in Group 1 with regard to any of the other parameters. There was also no significant difference in any parameter between the left

\section{Distance AP-SI}

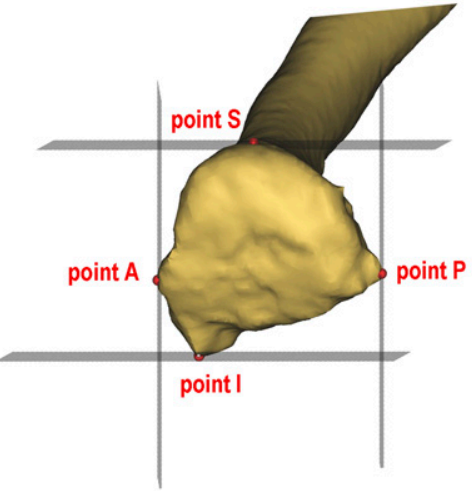

Symptomatic degenerative SC arthritis

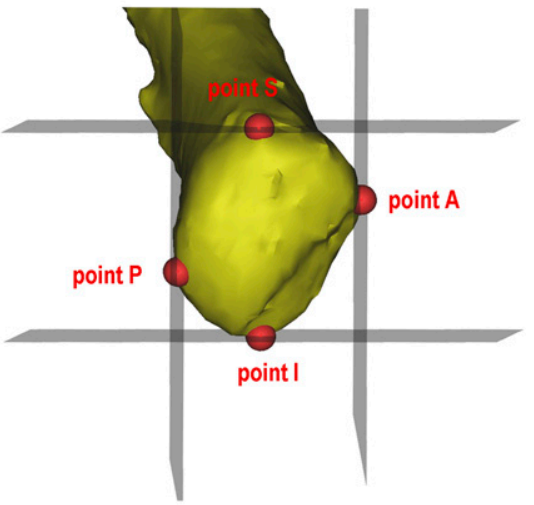

Normal SC

Fig. 4

Determination of points A, S, P, and I on the medial clavicular head. SC = sternoclavicular joint. 


\section{Distance frontal plane - point A}
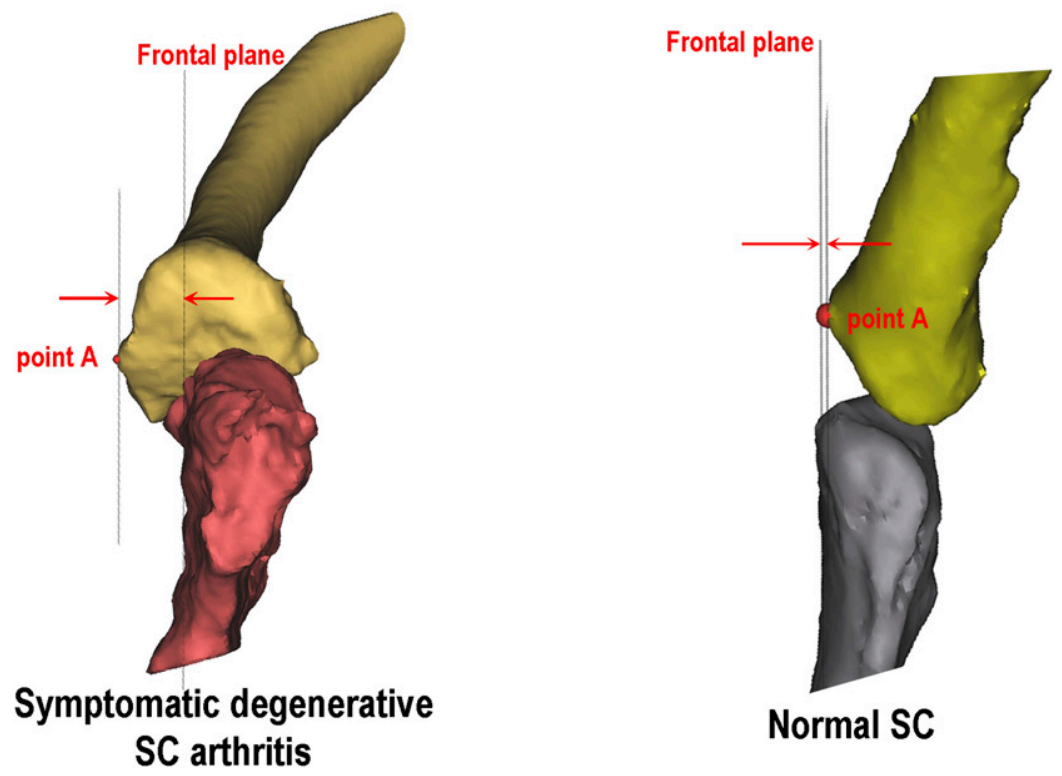

Fig. 5

Measurement of the distance between point $\mathrm{A}$ and the frontal plane. SC = sternoclavicular joint.

\section{Distance axial plane - point S}

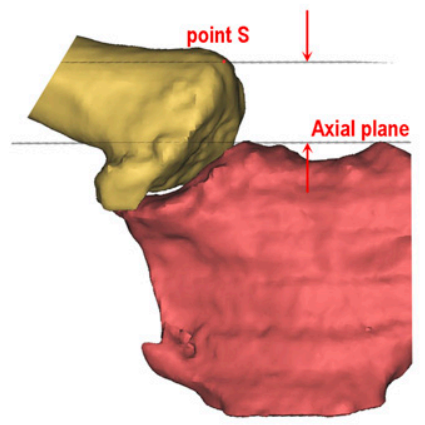

Symptomatic degenerative SC arthritis

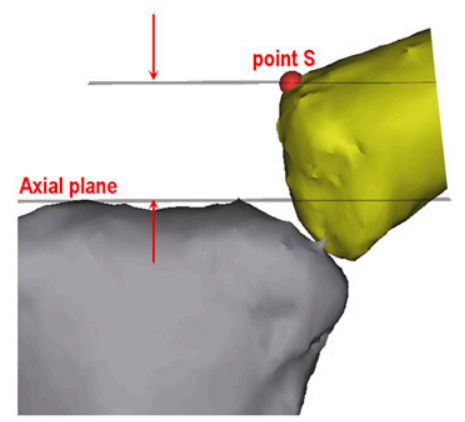

Normal SC

Fig. 6

Measurement of the distance between point $\mathrm{S}$ and the axial plane. SC = sternoclavicular joint.

and right sides ( $\mathrm{p}>0.2$ for all measurements). The AP distance was greater in men than in women on both the symptomatic and the contralateral side $(\mathrm{p}=0.002$ and $\mathrm{p}<0.001)$.

The AP distance of the clavicular head on the symptomatic, arthritic side was greater than the distance on the nonsymptomatic, contralateral side $(\mathrm{p}=0.004)$ and in the normal population $(\mathrm{p}<0.001)$. There was no significant difference in the AP distance between the non-symptomatic, contralateral side and the normal population $(\mathrm{p}=0.085)$. The SI distance of the clavicular head on the symptomatic, arthritic side was greater than that on the non-symptomatic, contralateral side $(\mathrm{p}=0.01)$ and compared with that in the normal population $(\mathrm{p}<0.001)$. There was no significant difference in the SI distance between the non-symptomatic, contralateral side and the normal population $(\mathrm{p}=0.279)$.

The AFr distance on the symptomatic, arthritic side was greater than the distance on the non-symptomatic, contralateral side $(\mathrm{p}<0.001)$ and in the normal population $(\mathrm{p}<0.001)$. The AFr distance was greater on the non-symptomatic, contralateral side than in the normal population $(\mathrm{p}<0.001)$.

The 95\% prediction interval of the AFr/AP ratio in the normal population was between 0.17 and -0.17 (mean, 0 ). 


\section{e109(5)}

The Journal of Bone \& Joint Surgery - JBjS. org Volume 96-A · Number $13 \cdot$ July 2, 2014
Relationship of Clavicular Head to Manubrium in Normal and Symptomatic Sternoclavicular JoInts

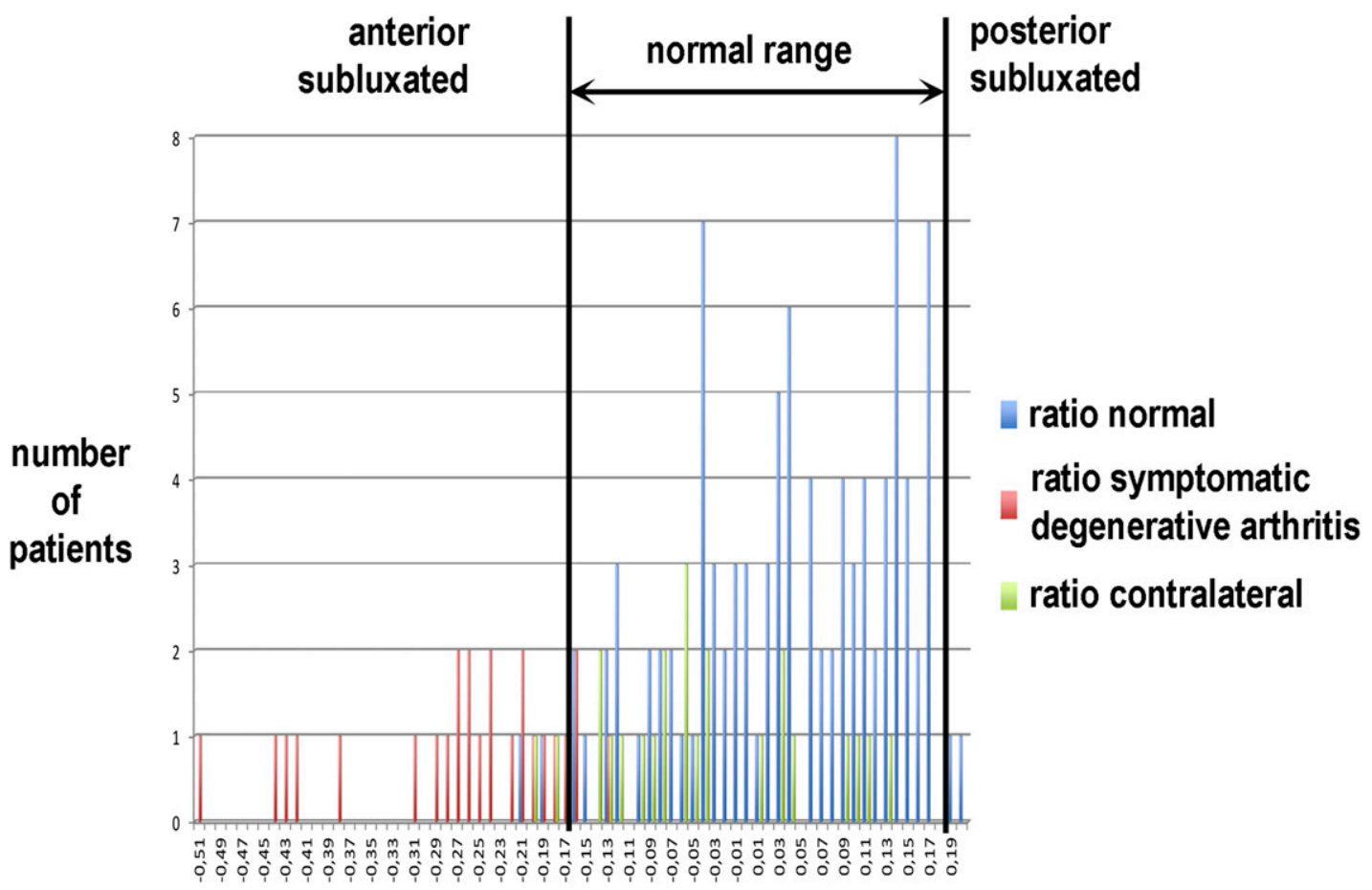

Fig. 7

Graph showing the AFr/AP ratios in all patients.

ratio AFr/AP

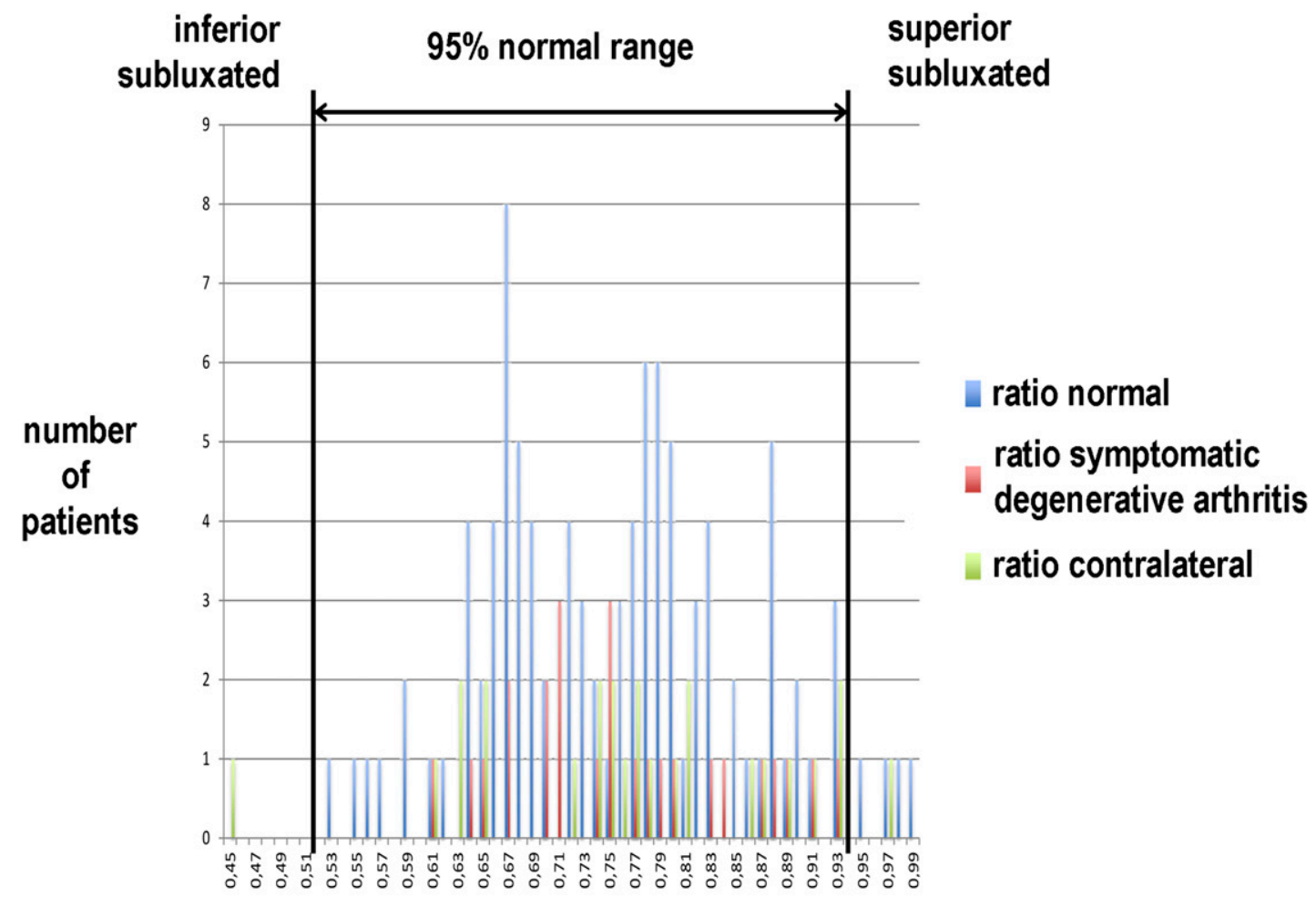

Fig. 8

Graph showing the SAx/SI ratios in all patients. 
The Journal of Bone \& Joint Surgery • Jbjs.org Volume 96-A · Number 13 · July 2, 2014
Relationship of Clavicular Head to Manubrium in Normal and Symptomatic Sternoclavicular Joints
Twenty-two of the twenty-five symptomatic patients had anterior subluxation, with a ratio between -0.13 and -0.51 , on the arthritic side. Two of the twenty-five patients had anterior subluxation on the non-symptomatic, contralateral side. The ratios on the nonsymptomatic, contralateral side ranged between 0.12 and -0.20 (Fig. 7).

The SAx distance was greater on the symptomatic arthritic side than in the normal population $(\mathrm{p}=0.0034)$. There was no significant difference between the SAx distance between the symptomatic, arthritic side and the non-symptomatic, contralateral side $(\mathrm{p}=0.219)$ or between the non-symptomatic, contralateral side and the normal population $(\mathrm{p}=0.551)$.

The $95 \%$ prediction interval of the SAx/SI ratio in the normal population was between 0.55 and 0.97 (mean, 0.76). No patient showed signs of superior subluxation on the arthritic side, with the ratios ranging between 0.64 and 0.93 . There were no cases of superior subluxation on the non-arthritic side of the symptomatic patients; the ratios ranged between 0.62 and 0.93 (Fig. 8).

\section{Discussion}

Cymptomatic sternoclavicular arthritis is not common. The swelling in patients with sternoclavicular arthritis has been described clinically as a subluxation. Two-dimensional CT evaluation of subluxation of the clavicle has been previously performed in patients with osteitis condensans but without quantification ${ }^{16}$. To our knowledge, we are the first to quantify subluxation in patients with symptomatic degenerative sternoclavicular arthritis.

The present study demonstrates that the 3D sternoclavicular relationship can be quantified reliably with low variability with use of 3D CT reconstruction in vivo.

The intraobserver and interobserver ICCs were excellent (0.93 and 0.9) for the definition of the best fitting plane of the bare area of the manubrium ${ }^{14}$.

Clinical examination of patients with symptomatic degenerative sternoclavicular arthritis sometimes reveals a prominent clavicle. This prominence may be due to the clavicle being anteriorly subluxated and/or a large clavicle. The width of the clavicle is significantly larger (by $4.2 \mathrm{~mm}$, on average) than that in the normal population, but this widening is not as great as the anterior displacement (8.4-mm difference, on average), indicating that anterior subluxation of the clavicle is clearly involved.

In the sagittal plane, the articular surface is, on average, directed $70^{\circ}$ inferiorly ${ }^{17}$; thus, a superior subluxation component would be suspected if there is anterior subluxation. Our study showed that, although there was a significant difference in the SAx distance between the arthritic side and the normal population, no patient had superior subluxation. A possible explanation is that the joint space had narrowed. Another possible explanation is erosion of the anterior surface of the manubrium, in which case the clavicle would not need to migrate superiorly to result in anterior migration.
The first-line treatment for symptomatic sternoclavicular arthritis is conservative, with nonsteroidal anti-inflammatory drugs, relative rest, or an intra-articular corticosteroid injection. Medial clavicle resection has been proposed if conservative treatment fails, and several techniques have been described ${ }^{18-23}$. The results of these techniques have been reported to be good in a few case reports ${ }^{24}$.

The clinical importance of this study lies in the fact that surgical treatment with medial clavicle resection will not correct the degenerative process of subluxation, but it is not known if this correction is necessary to obtain a good result.

This study has several weaknesses. We did not have an objective score for all symptomatic patients to allow correlation between the degree of subluxation and the clinical symptoms. Another weakness is our use of the asymptomatic sides as a control group and not a normal population of the same age. However, because there was no significant difference between the findings in the normal young population and those on the asymptomatic side, we believe that the values on the asymptomatic side can represent those in a normal population of the same age.

In conclusion, the morphology and position of the medial clavicular head relative to the manubrium can be evaluated adequately with the use of this newly developed reproducible measurement technique. Using this technique, we demonstrated that the clavicular prominence in patients with symptomatic sternoclavicular arthropathy is the result of both osseous enlargement and anterior subluxation of the clavicle.

\section{Appendix}

eA Tables showing the ICCs for intraobserver and interobeA server reliability as well as the mean distances for the different groups and parameters, and a figure demonstrating a manubrium held against a light, are available with the online version of this article as a data supplement at jbjs.org.

Note: The authors thank Wouter Huysse, MD, Department of Radiology, Ghent University Hospital; Stijn Vandamme, MD, Department of Radiology, AZ Groeninge Kortrijk; Jesse Marrannes, MD, Department of Radiology, Stedelijk Ziekenhuis Roeselare; Yves Fortems, Department of Orthopaedics and Traumatology, Sint-Augustinus Antwerp; Philippe Debeer, Department of Orthopaedics, University Hospital Leuven; Hans Van der Bracht, Department of Orthopaedics and Traumatology, Virga Jesse Hospital Hasselt, for their assistance with this work.

Alexander Van Tongel, MD

Jens Valcke, Msc

Iwein Piepers, Msc

Thomas Verschueren, MD

Lieven De Wilde, MD, PhD

Department of Orthopaedic Surgery and Traumatology,

Ghent University Hospital,

De Pintelaan 185,

9000 Ghent, Belgium.

E-mail address for A. Van Tongel: Alexander.vantongel@uzgent.be

\section{References}

1. Higginbotham TO, Kuhn JE. Atraumatic disorders of the sternoclavicular joint. J Am Acad Orthop Surg. 2005 Mar-Apr;13(2):138-45.
2. Robinson $\mathrm{CM}$, Jenkins PJ, Markham PE, Beggs I. Disorders of the sternoclavicular joint. J Bone Joint Surg Br. 2008 Jun;90(6):685-96. 


\section{$\operatorname{e109}(7)$}

The Journal of Bone \& Joint Surgery $\cdot$ JBjS. org Volume 96-A · Number 13 · July 2, 2014
Relationship of Clavicular Head to Manubrium in Normal and Symptomatic Sternoclavicular Joints
3. van Tongel A, MacDonald P, Leiter J, Pouliart N, Peeler J. A cadaveric study of the structural anatomy of the sternoclavicular joint. Clin Anat. 2012 Oct;25(7):903-10. Epub 2012 Jan 03.

4. Buckler WS. Primary generalized osteoarthritis with involvement of the sterno clavicular joints. Ann Phys Med. 1955 Jan;2(5):180-1.

5. Bremner RA. Monarticular, non-infective subacute arthritis of the sterno-clavlcular joint. J Bone Joint Surg Br. 1959 Nov;41:749-53.

6. Bonnin JG. Spontaneous subluxation of the sternoclavicular joint. Br Med J. 1960 Jul 23;2(5194):274-5.

7. Sadr B, Swann M. Spontaneous dislocation of the sterno-clavicular joint. Acta Orthop Scand. 1979 Jun;50(3):269-74.

8. Gerber C, Costouros JG, Sukthankar A, Fucentese SF. Static posterior humeral head subluxation and total shoulder arthroplasty. J Shoulder Elbow Surg. 2009 JulAug;18(4):505-10. Epub 2009 May 29.

9. Verstraeten TR, Deschepper E, Jacxsens M, Walravens S, De Coninck B, De Wilde LF. Operative guidelines for the reconstruction of the native glenoid plane: an anatomic three-dimensional computed tomography-scan reconstruction study. J Shoulder Elbow Surg. 2012 Nov;21(11):1565-72. Epub 2012 Jan 23.

10. Verstraeten TR, Deschepper E, Jacxsens M, Walravens S, De Coninck B, Pouliart N, De Wilde LF. Determination of a reference system for the three-dimensional study of the glenohumeral relationship. Skeletal Radiol. 2013 Aug;42(8):1061-71. Epub 2013 Jan 31.

11. Karelse AT, Bhatia DN, De Wilde LF. Prosthetic component relationship of the reverse Delta III total shoulder prosthesis in the transverse plane of the body.

J Shoulder Elbow Surg. 2008 Jul-Aug;17(4):602-7. Epub 2008 May 02.

12. Karelse A, Kegels L, De Wilde L. The pillars of the scapula. Clin Anat. 2007 May;20(4):392-9.

13. Anetzberger $\mathrm{H}$, Putz R. The scapula: principles of construction and stress. Acta Anat (Basel). 1996;156(1):70-80.
14. Xiu P, Shui D, Wang $Q$, Wang G, Lan Y. Anatomic and morphometric analysis of manubrium sterni as a source of autograft for anterior cervical fusion surgery using quantitative 3-dimensional computed tomographic scans. Spine (Phila Pa 1976). 2012 Jul 15;37(16):E935-41.

15. Shrout PE, Fleiss JL. Intraclass correlations: uses in assessing rater reliability. Psychol Bull. 1979 Mar;86(2):420-8.

16. van Holsbeeck M, van Melkebeke J, Dequeker J, Pennes DR. Radiographic findings of spontaneous subluxation of the sternoclavicular joint. Clin Rheumatol. 1992 Sep;11(3):376-81.

17. Wijeratna MD, Turmezei TD, Tytherleigh-Strong G. Novel assessment of the sternoclavicular joint with computed tomography for planning interventional approach. Skeletal Radiol. 2013 Apr;42(4):473-8. Epub 2012 Aug 30.

18. Rockwood CA Jr, Groh Gl, Wirth MA, Grassi FA. Resection arthroplasty of the sternoclavicular joint. J Bone Joint Surg Am. 1997 Mar;79(3):387-93.

19. Meis RC, Love RB, Keene JS, Orwin JF. Operative treatment of the painful sternoclavicular joint: a new technique using interpositional arthroplasty. J Shoulder Elbow Surg. 2006 Jan-Feb;15(1):60-6.

20. Pingsmann A, Patsalis T, Michiels I. Resection arthroplasty of the sternoclavicular joint for the treatment of primary degenerative sternoclavicular arthritis. J Bone Joint Surg Br. 2002 May;84(4):513-7.

21. Kuhn JE. Medial clavicle excision and sternoclavicular joint reconstruction. Williams GR, Ramsey ML, Wiesel SW, editors. Philadephia: Lippincott Wiliiams \& Wilkins; 2011. 22. Carrera EF, Archetti Neto N, Carvalho RL, Souza MA, Santos JB, Faloppa F. Resection of the medial end of the clavicle: an anatomic study. J Shoulder Elbow Surg. 2007 Jan-Feb;16(1):112-4. Epub 2006 Oct 19.

23. Bisson LJ, Dauphin N, Marzo JM. A safe zone for resection of the medial end of the clavicle. J Shoulder Elbow Surg. 2003 Nov-Dec;12(6):592-4.

24. Acus RW 3rd, Bell RH, Fisher DL. Proximal clavicle excision: an analysis of results. J Shoulder Elbow Surg. 1995 May-Jun;4(3):182-7. 